\title{
Fatores associados à adesão a grupos de atividades físicas na atenção básica
}

\author{
Factors associated with adherence to physical activity groups in primary health care \\ Factores asociados a la adherencia a grupos de actividad física en la atención primaria \\ Anderson Martins Silva', Daniele Sirineu Pereira², Maria Geracina de Souza ${ }^{3}$, Darlene Graciele Carvalho4, \\ Isabella Tirado Freire Lopes ${ }^{5}$, Silvia Lanziotti Azevedo da Silva ${ }^{6}$
}

RESUMOIO objetivo do estudo foi verificaraadesão deusuários a grupos de atividade física realizados por fisioterapeutas na atenção básica (AB) e seus fatores associados. Quanto à metodologia, trata-se de estudo transversal, quantitativo. Foi aplicado um questionário semiestruturado e outro de adesão adaptado, que investiga fatores gerais, motivadores e barreiras que podem influenciar na adesão. A amostra foi subdivida em 3 grupos em relação à adesão: 1-30\% de frequência em 6 meses baixa adesão, de 31 - 70\% média e acima de 71\% alta adesão. Os grupos foram comparados pelos testes de Kruskall-Wallis e análise de variância (Anova) e a associação avaliada por regressão logística multinomial. Nos resultados, em relação à adesão, 46,8\% da amostra apresentou baixa, 42,2\% média e $11 \%$ alta adesão entre os 154 participantes, com média

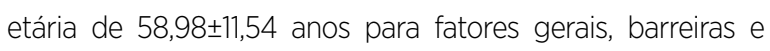
motivadores foram diferentes $(p<0,05)$ entre os grupos, e menor relato de fatores gerais de não adesão reduziram a chance de média adesão aos grupos. O maior tempo de participação nos grupos aumentou a chance de média e alta adesão em toda a amostra. Concluímos que o conhecimento dos fatores que influenciam positivamente na adesão, como tempo de participação, disposição e interesse, facilitam o desenvolvimento de estratégias pelos profissionais da $A B$ para sua manutenção. Os resultados mostraram que existem fatores que interferem na adesão aos grupos realizados na $A B$. Descritores | Atividade Motora; Atenção Primária à Saúde; Estratégia de Saúde da Família; Fisioterapia.
ABSTRACT I This study aimed to verify the users' adherence to physical activity groups conducted by physical therapists at primary health care (PHC) and their related factors. This is a cross-sectional quantitative study. A semi-structured questionnaire and an adapted adherence questionnaire were applied, which investigate general factors, motivators, and obstacles that can affect adherence. The sample was divided into three groups according to adherence: 1-30\% of frequency in 6 months as low adherence; 31 to $70 \%$ as medium adherence; and above $71 \%$ as high adherence. The groups were compared by Kruskal-Wallis and ANOVA tests, and the association was evaluated by multinomial logistic regression. Regarding adherence results, $46.8 \%$ of the sample had low, 42.2\% medium, and 11\% high adherence among the 154 participants, with a mean age of $58.98 \pm 11.54$ years for general factors, barriers and motivators were different ( $p<0.05$ ) between groups, and lower reporting of general nonadherence factors reduced the chance of medium adherence to groups. The longer participation time in the groups increased the chance of average and high adherence across the sample. We conclude that the knowledge of the factors that positively affect adherence, such as time of participation, willingness, and interest, facilitate the development of strategies by PHC professionals for its maintenance. The results showed that there are factors that affect the adherence of groups in $\mathrm{PHC}$. Keywords I Motor Activity; Primary Health Care; Family Health Strategy; Physical Therapy.

Estudo realizado nas Unidades Básicas Saúde da Família Caensa, Boa Esperança, Pinheirinho/Santa Clara e Santos Reis, do município de Alfenas(MG), com aprovação da Secretaria Municipal de Saúde.

Universidade Federal de Alfenas (Unifal) - Alfenas (MG), Brasil. E-mail: anderson.fisio@yahoo.com.br. Orcid: 0000-0003-4092-5537

2Universidade Federal de Minas Gerais (UFMG) - Belo Horizonte (Brasil). E-mail: daniele.sirineu@gmail.com. Orcid: 0000-0002-4868-9244

3 Universidade Federal de Alfenas (Unifal) - Alfenas (MG), Brasil. E-mail: maria.geracina.souza@gmail.com. Orcid: 0000-0003-0890-3848

${ }^{4}$ Universidade Federal de Alfenas (Unifal) - Alfenas (MG), Brasil. E-mail: darlene.graciele@hotmail.com. Orcid: 0000-0001-8033-1421

${ }^{5}$ Universidade Federal de Alfenas (Unifal) - Alfenas (MG), Brasil. E-mail: isabellatiradofreire@gmail.com. Orcid: 0000-0002-1610-1565

${ }^{6}$ Universidade Federal de Juiz de Fora (UFJF) - Juiz de Fora, Brasil. E-mail: silviafisiojf@yahoo.com.br. Orcid: 0000-0002-2323-2029 
RESUMEN I El objetivo de este estudio fue verificar la adherencia de los usuarios a los grupos de actividad física llevada a cabo por fisioterapeutas en la atención primaria (AP) y sus factores asociados. En cuanto al método, se trata de un estudio transversal, cuantitativo. Se aplicó un cuestionario semiestructurado y un cuestionario de adherencia adaptado, que examina os factores generales, los motivadores y las barreras que pueden influir en la adherencia al grupo. La muestra se subdividió en 3 grupos en relación a la adherencia: del 1-30\% de frecuencia en 6 meses baja adherencia, entre el 31-70\% mediana adherencia y superior al 71\% alta adherencia. Los grupos se compararon mediante las pruebas de Kruskal-Wallis y el análisis de varianza (Anova), y la asociación se evaluó mediante la regresión logística multinomial. Los resultados evidenciaron que, en relación a la adherencia, el 46,8\% de la muestra tuvo baja adherencia, el
42,2\% mediana y el 11\% alta entre los 154 participantes con una edad promedio de 58,98 $\pm 11,54$ años para factores generales, las barreras y los motivadores fueron distintos $(p<0,05)$ entre los grupos, y un menor relato de los factores generales de la no adherencia redujo la posibilidad de tener una mediana adherencia a los grupos. El mayor tiempo de participación en los grupos aumentó la posibilidad de tener una adherencia mediana y alta en la muestra. Se concluye que el conocimiento de los factores que influyen positivamente en la adherencia, como el tiempo de participación, la disposición y el interés, facilitan el desarrollo de estrategias por parte de los profesionales de AP para el mantenimiento en el grupo. Los resultados mostraron que existen factores que interfieren en la adherencia a los grupos en la AP. Palabras clave I Actividad Motora; Atención Primaria de Salud; Estrategia de Salud Familiar; Fisioterapia.

\section{INTRODUÇÃO}

A atenção básica $(\mathrm{AB})$ é um conjunto de ações de saúde direcionadas ao desenvolvimento da atenção integral ${ }^{1}$. Em busca desse objetivo são realizados grupos operativos, como aqueles que desenvolvem atividades físicas e educativas, coordenados por fisioterapeutas ${ }^{2,3}$.

A inatividade física é responsável por cerca de $80 \%$ das principais doenças crônicas ${ }^{4,5}$. Reconhecer a importância da atividade física para a saúde é importante, bem como identificar os fatores motivadores de uma prática regular, além de buscar estratégias que aumentem a adesão $0^{6,7}$.

O conceito mais utilizado considera adesão a um programa de exercícios como a razão entre o número de sessões realizadas e ofertadas ${ }^{8,9}$. A taxa média de adesão encontrada em uma amostra de 27.343 pessoas acima de 18 anos foi $65,2 \%$, e a taxa média de sedentarismo foi $18,8 \%{ }^{10}$. Entre os indivíduos que iniciam um programa de atividade física, $50 \%$ interrompe em até 6 meses $^{11}$.

Uma vez que a $A B$ oferece grupos para prática de atividade física regular nas Unidades Básicas de Saúde da Família (UBSF), torna-se importante avaliar sua adesão às propostas oferecidas. O objetivo deste estudo foi verificar a adesão de usuários a esses grupos, organizados por fisioterapeutas, e verificar fatores associados a ela.

\section{METODOLOGIA}

Estudo transversal quantitativo, aprovado pelo Comitê de Ética em Pesquisa da Universidade Federal de
Alfenas (Unifal-MG), sob o parecer no 1.679.885. Todos os participantes assinaram Termo de Consentimento Livre e Esclarecido.

A amostra foi de conveniência, composta por usuários participantes dos grupos para prática de atividade física realizados por fisioterapeutas em 4 UBSF de Alfenas, Minas Gerais. Foram incluídos todos que concordaram em participar e excluídos aqueles que relataram não poder ou querer comparecer à entrevista. As entrevistas aconteceram entre junho e dezembro de 2016, nas UBSF, em horário combinado com os participantes.

Os exercícios dos grupos tinham duração de uma hora, divididos em alongamento, fortalecimento e coordenação global. A adesão foi mensurada pelo percentual de presenças nos grupos por seis meses. A amostra foi então dividida em três grupos, considerando alta adesão acima de $71 \%$ de presença, média adesão entre $31 \%$ e $70 \%$ e baixa adesão abaixo de $30 \%$, determinados por tercil, garantindo maior equivalência do percentual de frequência na atividade por grupo.

A caracterização da amostra foi realizada por questionário semiestruturado composto por dados sociodemográficos, uso de serviços de fisioterapia, variáveis clínicas (número de comorbidades e medicamentos), tabagismo, etilismo, tempo de participação nos grupos e relato de dor.

Para verificação do nível de adesão aos grupos foi utilizado questionário adaptado de Picorelli et al. ${ }^{12}$, que relaciona motivos gerais que levam o indivíduo à faltar ao grupo (falta de tempo, disposição, interesse), motivadores que incentivam a participação (benefícios dos exercícios) e barreiras que levam à não participação (insatisfação com os exercícios). $\mathrm{O}$ instrumento foi aplicado por um examinador treinado. 


\section{Análise estatística}

Para descrição da amostra, foram calculados valores de média, desvio-padrão e valores máximos e mínimos para as variáveis contínuas e percentuais para as categóricas. A comparação entre os grupos para as covariáveis foi realizada pelo teste de Kruskall-Wallis para variáveis categóricas e contínuas com distribuição não normal, e testes Anova para variáveis contínuas com distribuição normal. A normalidade dos dados foi testada pelo teste de Kolmogorov-Smirnov.

Para análise do questionário de adesão, em cada bloco (geral, motivadores e barreiras) foi atribuído 1 ponto para as respostas "sim" e 0 para "não", e uma soma final em cada bloco. As somas das respostas dadas pelos usuários em cada bloco foram comparadas entre os grupos pelo teste de Kruskall-Wallis.
Para verificação dos fatores relatados pelos usuários associados a adesão, foi feita análise de regressão logística multinomial, sendo inseridas no modelo ajustado covariáveis com valor $\mathrm{p}<0,20$ na análise univariada. A categoria "baixa adesão" foi considerada referência.

As análises foram realizadas no software estatístico $R$ versão 3.4.1 e considerado nível de significância $p \leqq 0,05$.

\section{RESULTADOS}

A amostra foi composta por 154 usuários, 46,8\% apresentaram baixa adesão, 42,2\% média adesão e $11 \%$ alta adesão ao grupo. A maioria (90,9\%) era composta por mulheres que sabiam ler e escrever (89,6\%) e não fazem fisioterapia atualmente $(94,8 \%)$. Demais características sociodemográficas estão descritas na Tabela 1.

Tabela 1. Caracterização da amostra em relação a dados sociodemográficos e uso dos serviços de saúde (n=154)

\begin{tabular}{|c|c|c|c|}
\hline Variável & $n / \%$ & média \pm desvio-padrão & valor máximo/mínimo \\
\hline \multicolumn{4}{|l|}{ Sexo } \\
\hline Feminino & $140(90,9)$ & & \\
\hline Masculino & $14(9,1)$ & & \\
\hline Idade & & $58,98( \pm 11,5)$ & $91 / 27$ \\
\hline \multicolumn{4}{|l|}{ Estado Civil } \\
\hline Vive com companheiro & $91(59,1)$ & & \\
\hline Não vive com companheiro & $63(40,9)$ & & \\
\hline \multicolumn{4}{|l|}{ Cor ou Raça } \\
\hline Branca & $82(53,2)$ & & \\
\hline Não branca & $72(46,8)$ & & \\
\hline \multicolumn{4}{|l|}{ Ler/Escrever } \\
\hline Sim & $138(89,6)$ & & \\
\hline Não & $16(10,4)$ & & \\
\hline Anos de escolaridade & & $9,60( \pm 15,3)$ & $13 / 1$ \\
\hline \multicolumn{4}{|l|}{ Tem filhos } \\
\hline Sim & $133(86,4)$ & & \\
\hline Não & $21(13,6)$ & & \\
\hline número de filhos & & $3,17( \pm 1,8)$ & $10 / 1$ \\
\hline \multicolumn{4}{|l|}{ Mora sozinho } \\
\hline $\operatorname{sim}$ & $24(15,6)$ & & \\
\hline Não & $130(84,4)$ & & \\
\hline \multicolumn{4}{|l|}{ Atividade remunerada } \\
\hline Sim & $51(33,1)$ & & \\
\hline Não & $103(66,9)$ & & \\
\hline \multicolumn{4}{|l|}{ Faz fisioterapia atualmente } \\
\hline Sim & $8(5,2)$ & & \\
\hline Não & $146(94,8)$ & & \\
\hline \multicolumn{4}{|l|}{ Já fez fisioterapia } \\
\hline Sim & $65(42,5)$ & & \\
\hline Não & $88(57,5)$ & & \\
\hline
\end{tabular}


O tempo médio de participação no grupo foi 32,88 $( \pm 37,1)$ meses e $69,5 \%$ da amostra relatava dor, sendo esta crônica em $94,4 \%$ dos relatos. Demais características clínicas e hábitos de vida estão inseridos na Tabela 2 .

Os grupos, divididos pela adesão, apresentaram médias de idade e tempo de participação diferentes entre si $(\mathrm{p}<0,05)$ (Tabela 3).

A comparação das médias de cada bloco do questionário de adesão entre os grupos de baixa, média e alta adesão foi diferente para o bloco "geral" e "motivadores" $(p<0,05)$ (Tabela 4).
A análise de regressão logística multinomial mostrou que indivíduos que relataram menos motivos gerais para não adesão tiveram menos chance de ter média adesão, no modelo bruto (OR=0,82,IC95\%0,70-0,97) e ajustado (OR=0,75,IC95\%0,59-0,95). Em relação ao tempo de participação, indivíduos com mais tempo de participação apresentaram mais chance de média $(\mathrm{OR}=1,02, \mathrm{IC} 95 \% 1,01-1,05)$ e alta (OR=1,04,IC95\%1,01-1,07) adesão (Tabela 5).

Tabela 2. Descrição da amostra em relação a variáveis clínicas, tabagismo, etilismo, tempo de participação no grupo e relato de dor (n=154)

\begin{tabular}{|c|c|c|c|}
\hline Variável & $n / \%$ & média ( \pm desvio-padrão) & valor máximo/mínimo \\
\hline Número de comorbidades & & $2,03( \pm 1,2)$ & $7 / 0$ \\
\hline Número de medicamentos utilizados & & $3,64( \pm 2,4)$ & $12 / 0$ \\
\hline \multicolumn{4}{|l|}{ Hábito de fumar } \\
\hline Nunca fumou & $96(62,3)$ & & \\
\hline Fuma/já fumou & $58(37,7)$ & & \\
\hline \multicolumn{4}{|l|}{ Consumo de álcool } \\
\hline Não consome & $78(50,6)$ & & \\
\hline Consome qualquer quantidade & $76(49,4)$ & & \\
\hline Tempo de participação no grupo & & $32,88( \pm 37,1)$ & $1 / 240$ \\
\hline \multicolumn{4}{|l|}{ Dor } \\
\hline Sim & $107(69,5)$ & & \\
\hline Não & $47(30,5)$ & & \\
\hline \multicolumn{4}{|l|}{ Caracterização da dor } \\
\hline Agudo & $6(5,6)$ & & \\
\hline Crônico & $101(94,4)$ & & \\
\hline Intensidade da dor"* & & $6,91( \pm 2,5)$ & $1 / 10$ \\
\hline
\end{tabular}

"Meses; "escala visual analógica (EVA).

Tabela 3. Comparação entre as variáveis sociodemográficas, clínicas, caracterização dos grupos e uso dos serviços de saúde em relação aos grupos de adesão ( $n=154)$

\begin{tabular}{|c|c|c|c|c|}
\hline \multirow{3}{*}{ Variável } & \multicolumn{4}{|c|}{ Valores } \\
\hline & \multicolumn{4}{|c|}{ Adesão } \\
\hline & $\operatorname{Baixa}(72)(n / \%)$ & Média(65) (n/\%) & Alta(17) $(n / \%)$ & $\mathbf{P}$ \\
\hline \multicolumn{5}{|l|}{ Variáveis sociodemográficas } \\
\hline \multicolumn{5}{|l|}{$\operatorname{Sexo}^{1}(n / \%)$} \\
\hline Feminino & $69(95,8)$ & $56(86,1)$ & $15(88,2)$ & $0,13^{*}$ \\
\hline Masculino & $3(4,1)$ & $9(13,8)$ & $2(11,7)$ & \\
\hline Idade² (média \pm desvio-padrão) & $56,9( \pm 10,7)$ & $60,49( \pm 12,1)$ & $62( \pm 11,7)$ & $0,09 *$ \\
\hline \multicolumn{5}{|l|}{ Estado civil' (n/\%) } \\
\hline Vive com o companheiro & $47(65,2)$ & $37(56,9)$ & $7(41,1)$ & $0,17^{* *}$ \\
\hline Não vive com o companheiro & $25(34,8)$ & $28(43,1)$ & $10(58,9)$ & \\
\hline \multicolumn{5}{|l|}{$\operatorname{Cor}^{1}(n / \%)$} \\
\hline Branca & $36(50,0)$ & $37(56,9)$ & $9(52,9)$ & 0,72 \\
\hline Não branca & $36(50,0)$ & $28(43,1)$ & $8(47,1)$ & \\
\hline
\end{tabular}


Tabela 3. Continuação

\begin{tabular}{|c|c|c|c|c|}
\hline \multirow{3}{*}{ Variável } & \multicolumn{4}{|c|}{ Valores } \\
\hline & \multicolumn{4}{|c|}{ Adesão } \\
\hline & Baixa(72) (n/\%) & Média(65) (n/\%) & Alta(17) $(n / \%)$ & $\mathbf{P}$ \\
\hline \multicolumn{5}{|l|}{ Ler/escrever ${ }^{1}(n / \%)$} \\
\hline Sim & $63(87,5)$ & $60(92,3)$ & $15(88,2)$ & 0,64 \\
\hline Não & $9(12,5)$ & $5(7,7)$ & $2(11,8)$ & \\
\hline Anos de escolaridade² (média \pm desvio-padrão) & $12,90( \pm 21,5)$ & $7,05( \pm 4,2)$ & $5,35( \pm 3,9)$ & $0,03^{*}$ \\
\hline \multicolumn{5}{|l|}{ Tem filhos ${ }^{1}$} \\
\hline $\operatorname{Sim}$ & $63(87,5)$ & $56(86,1)$ & $14(82,3)$ & 0,85 \\
\hline Não & $9(12,5)$ & $9(13,9)$ & $3(17,7)$ & \\
\hline № de filhos² (média \pm desvio-padrão) & $3,15( \pm 1,5)$ & $3,23( \pm 2,1)$ & $3( \pm 2,1)$ & 0,91 \\
\hline \multicolumn{5}{|l|}{ Atividade remunerada' (n/\%) } \\
\hline Sim & $26(36,1)$ & $22(33,8)$ & $4(23,5)$ & 0,34 \\
\hline Não & $46(63,9)$ & $42(66,2)$ & $14(76,5)$ & \\
\hline \multicolumn{5}{|l|}{ Faz fisioterapia' (n/\%) } \\
\hline Sim & $3(4,1)$ & $4(6,1)$ & $1(5,8)$ & 0,86 \\
\hline Não & $69(95,9)$ & $61(93,9)$ & $16(94,2)$ & \\
\hline \multicolumn{5}{|l|}{ Já fez Fisioterapia'(n/\%) } \\
\hline Sim & $26(31,1)$ & $31(47,6)$ & $8(47)$ & 0,32 \\
\hline Não & $46(63,9)$ & $33(52,4)$ & $9(53)$ & \\
\hline Número de comorbidades² (média \pm desvio-padrão) & $2,02( \pm 1,2)$ & $3,87( \pm 2,5)$ & $2,85( \pm 1,7)$ & 0,36 \\
\hline Número de medicamentos² (média \pm desvio-padrão) & $3,58( \pm 2,3)$ & $3,87( \pm 2,5)$ & $2,86( \pm 1,7)$ & 0,36 \\
\hline \multicolumn{5}{|l|}{ Hábito de fumar' (n/\%) } \\
\hline Fuma/já fumou & $43(59,7)$ & $43(66,1)$ & $10(58,8)$ & 0,70 \\
\hline Não fuma & $29(40,3)$ & $22(33,9)$ & $7(41,2)$ & \\
\hline \multicolumn{5}{|l|}{ Consumo de alcool' ( $\mathrm{n} / \%)$} \\
\hline Não consome & $34(47,2)$ & $37(56,9)$ & $7(41,1)$ & 0,37 \\
\hline Consome qualquer quantidade & $38(58,2)$ & $28(43,1)$ & $10(58,9)$ & \\
\hline \multicolumn{5}{|l|}{$\operatorname{Dor}^{1}(n / \%)$} \\
\hline Sim & $54(75)$ & $43(66,1)$ & $10(58,8)$ & 0,32 \\
\hline Não & $18(25)$ & $22(33,9)$ & $7(41,2)$ & \\
\hline \multicolumn{5}{|l|}{ Caracterização da dor' (n/\%) } \\
\hline Aguda & $2(2,7)$ & $3(4,6)$ & $1(5,8)$ & 0,64 \\
\hline Crônica & $52(97,3)$ & $40(95,4)$ & $9(94,2)$ & \\
\hline Intensidade da dor (EVA)² (média „desvio-padrão) & $6,89( \pm 2,6)$ & $7,23( \pm 2,4)$ & $5,60( \pm 2)$ & $0,18 *$ \\
\hline Tempo de participação² (média \pm desvio-padrão) & $25,87( \pm 36,3)$ & $37,11( \pm 35,5)$ & $46,35( \pm 42,1)$ & $0,04^{\circ}$ \\
\hline
\end{tabular}

'Teste de Kruskall-Wallis; ${ }^{2}$ Anova; " $p<0,05$ : significativo; " $p<0,20$ : covariável do modelo de regressão.

Tabela 4. Comparação dos fatores relacionados a adesão por blocos pelos usuários entre os grupos de baixa, média e alta adesão (n=154)

\begin{tabular}{|c|c|c|c|c|}
\hline \multirow{3}{*}{ Questionário } & \multicolumn{4}{|c|}{ 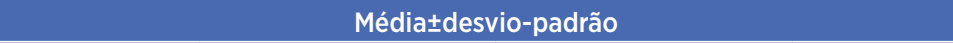 } \\
\hline & \multicolumn{4}{|c|}{ Adesão } \\
\hline & $\begin{array}{c}\text { Baixa } \\
(n=72)\end{array}$ & $\begin{array}{l}\text { Média } \\
(n=65)\end{array}$ & $\begin{array}{c}\text { Alta } \\
(n=17)\end{array}$ & p \\
\hline Geral & $\begin{array}{r}3,9 \\
( \pm 2,8)\end{array}$ & $\begin{array}{r}2,8 \\
( \pm 1,8)\end{array}$ & $\begin{array}{r}2,6 \\
( \pm 2,4)\end{array}$ & $0,01^{\circ}$ \\
\hline Motivadores & $\begin{array}{r}10,7 \\
( \pm 1,2)\end{array}$ & $\begin{array}{r}11,1 \\
( \pm 0,9)\end{array}$ & $\begin{array}{l}11,4 \\
( \pm 1)\end{array}$ & $0,02^{\circ}$ \\
\hline Barreiras & $\begin{array}{r}3,8 \\
( \pm 2,2)\end{array}$ & $\begin{array}{r}3,4 \\
( \pm 1,9)\end{array}$ & $\begin{array}{r}2,6 \\
( \pm 1,5)\end{array}$ & $0,07^{*}$ \\
\hline
\end{tabular}

${ }^{* *} p<0,20$ : entra no modelo de regressão logística; ${ }^{*} p<0,05$ : significativo. 
Tabela 5. Análise de Regressão Logística Multinomial para determinação de associações com adesão aos grupos.

\begin{tabular}{|c|c|c|c|c|c|}
\hline \multirow{2}{*}{ Adesão* } & \multirow{2}{*}{ Variável } & \multicolumn{2}{|c|}{ Modelo Bruto } & \multicolumn{2}{|c|}{ Modelo Ajustado } \\
\hline & & Odds Ratio & IC95\% & Odds Ratio & IC95\% \\
\hline \multirow{13}{*}{ Média Adesão } & Motivos Gerais & 0,82 & $0,70-0,97^{\circ}$ & 0,75 & $0,59-0,95^{\circ}$ \\
\hline & Motivadores & 1,35 & $0,98-1,86$ & 1,06 & $0,65-1,72$ \\
\hline & Barreiras & 0,98 & $0,82-1,17$ & 0,85 & $0,66-1,08$ \\
\hline & Idade & & & 0,99 & $0,94-1,04$ \\
\hline & Sexo & & & & \\
\hline & Feminino & & & Ref & Ref \\
\hline & Masculino & & & 6,12 & $0,83-44,9$ \\
\hline & Estado Civil & & & & \\
\hline & Vive com o companheiro & & & Ref & Ref \\
\hline & Não vive com o companheiro & & & 1,95 & $0,63-5,97$ \\
\hline & Anos de Escolaridade & & & 0,95 & $0,90-1,02$ \\
\hline & EVA & & & 1,20 & $0,97-1,47$ \\
\hline & Tempo de Participação & & & 1,02 & $1,01-1,05^{\circ}$ \\
\hline \multirow{13}{*}{ Alta adesão } & Motivos Gerais & 0,83 & $0,63-1,09$ & 0,79 & $0,52-1,20$ \\
\hline & Motivadores & 1,89 & $0,98-3,70$ & 0,79 & $0,31-1,98$ \\
\hline & Barreiras & 0,81 & $0,59-1,11$ & 0,66 & $0,39-1,14$ \\
\hline & Idade & & & 0,95 & $0,86-1,05$ \\
\hline & Sexo & & & & \\
\hline & Feminino & & & Ref & Ref \\
\hline & Masculino & & & 63,41 & $0,41-166,54$ \\
\hline & Estado Civil & & & & \\
\hline & Vive com o companheiro & & & Ref & Ref \\
\hline & Não vive com o companheiro & & & 3,39 & $0,40-28,4$ \\
\hline & Anos de Escolaridade & & & 0,70 & $0,50-1,01$ \\
\hline & EVA & & & 0,89 & $0,60-1,31$ \\
\hline & Tempo de Participação & & & 1,04 & $1,01-1,07^{\circ}$ \\
\hline
\end{tabular}

'Média Adesão: 31-70\% de presença, alta adesão: acima de 71\% de presença; Categoria de referência adesão: baixa adesão: 0 -30\% de presença; EVA: Escala Visual Analógica.

\section{DISCUSSÃO}

No presente estudo, entre os participantes dos grupos avaliados, mais tempo de participação aumentou as chances de média e alta adesão e relatar mais motivos gerais para não adesão diminuiu a chance de média adesão. A amostra deste estudo foi semelhante a de um estudo realizado com 17 indivíduos participantes de um programa de atividade física em uma UBSF de Botucatu, São Paulo, onde $82 \%$ eram mulheres, com média de idade $67( \pm 6,17)$ anos, $65 \%$ viviam com companheiros e a média de tempo no programa foi de $7,75( \pm 6,32)$ anos $^{13}$. Grande parte dos participantes deste estudo são mulheres, confirmando os dados encontrados na literatura ${ }^{14,15}$. Um estudo realizado em Pelotas, Rio Grande do Sul, contou com 195 mulheres e apenas quatro homens ${ }^{14}$. Tal diferença de gênero é observada em estudos realizados em
Florianópolis, Santa Catarina, com grupos de convivência, nos quais $79 \%$ e $91 \%$ dos participantes são mulheres ${ }^{15,16}$. Essa diferença pode ser explicada pelas oportunidades de lazer diferenciadas, pela percepção dos exercícios oferecidos nas UBSF como pouco exigentes fisicamente e pela preocupação inferior dos homens com a prevenção, por questões culturais e laborais ${ }^{17,18}$.

Quando analisada a adesão, em um estudo realizado com 113 mulheres idosas comunitárias de Belo Horizonte, Minas Gerais, submetidas a um programa de exercícios domiciliares, com supervisão indireta, $26,64 \%$ foram consideradas aderentes e $73,4 \%$ não aderentes, taxa de adesão superior à encontrada nesse estudo ${ }^{12}$. Essa diferença pode ser devida à supervisão indireta e a realização no domicílio, em qualquer horário, e ao menor tempo de observação, o que pode minimizar a constatação da não adesão após período mais prolongado. 
Existem fatores que influenciam na adesão aos programas de atividade física de forma positiva e negativa ${ }^{19}$. Este estudo identificou que maior relato de motivos gerais para não adesão diminuiu a chance de média adesão. Um estudo realizado no Recife, Pernambuco, com 120 idosos participantes há pelo menos de seis meses em programas sociais voltados para a saúde e qualidade de vida, realizados em espaços públicos, não encontrou associação entre motivos gerais e não adesão aos programas avaliados ${ }^{20}$.Tal diferença pode ocorrer porque os exercícios são mais diversificados e considerados referência no município, apresentado assim maior adesão e menos faltas. Outro ponto importante é o tempo de participação no programa em questão, que é sabido que ajuda na redução da chance de baixa adesão.

Apesar de menos frequentes no grupo de baixa adesão, o relato de fatores motivadores não aumentou a chance de apresentar média ou alta adesão. Os motivadores foram considerados importantes para maior adesão em um estudo em Botucatu, São Paulo, que encontrou aproximadamente $50 \%$ de adesão entre 17 participantes de realizado na $\mathrm{AB}^{13}$. O resultado diferenciado pode ser devido a perfis diferenciados de usuários, uma vez que o presente estudo analisou quatro unidades diferentes, o que gera maior variabilidade de respostas. $\mathrm{O}$ relato das barreiras foi associado à adesão. Um estudo realizado com 113 mulheres idosas da comunidade de Belo Horizonte, Minas Gerais, encontrou associação entre barreiras e baixa adesão. Entretanto, este estudo analisou atividades domiciliares orientadas e com supervisão indireta, tipo de atividades que exigem muito mais disciplina em sua realização ${ }^{12}$. O presente estudo analisou grupos em UBSF, com supervisão direta e grande interação social, o que pode minimizar a importância das barreiras no prejuízo da adesão.

Participantes com maior tempo de participação tiveram mais chance de apresentar média e alta adesão. No estudo realizado com 199 idosos em Pelotas, Rio Grande do Sul, cuja adesão foi superior a deste estudo, a maior parte dos participantes estava no programa de atividades físicas havia no mínimo seis meses e no máximo cinco anos ${ }^{14}$, o que fortalece a evidência relacionada ao tempo de participação influenciar a adesão. Com o aumento do tempo de prática das atividades, os benefícios psicológicos sobrepõem os estéticos e, provavelmente por esta razão, os indivíduos apresentam maior adesão, identificando a atividade física como prazerosa ${ }^{21}$.

Uma limitação do estudo foi o foco dos grupos avaliados ser prevenção e promoção de saúde. Tais atividades podem ser menos estimulantes que atividades curativas e, por isso, a adesão ser menor e sua avaliação mais difícil ${ }^{22}$. Um ponto forte do estudo foi seu tempo de duração, ideal para avaliar a adesão. Segundo a literatura 50\% da população que inicia um programa de exercícios o interrompe em até 6 meses $^{11}$, e o tempo de acompanhamento foi capaz de perceber tais desistências e investigar suas razões. Outro ponto importante a ser destacado é a amostra composta por usuários participantes de grupos oferecidos em UBSF, universo pouco explorado em estudos na fisioterapia.

\section{CONCLUSÃO}

Em conjunto com os demais profissionais das UBSF, os fisioterapeutas devem buscar aumentar sempre o vínculo com o usuário, evitar desistências precoces e criar estratégias para aumentar a adesão aos grupos, sempre voltados para aumento dos benefícios da promoção de saúde e prevenção de agravos.

\section{REFERÊNCIAS}

1. Brasil. Ministério da Saúde. Política Nacional de Atenção Básica [Internet]. Brasília, DF: 2012 [cited 2020 Nov 3]. Available from: https://dab.saude.gov.br/portaldab/biblioteca. php?conteudo=publicacoes/pnab.

2. Augusto VG, Aquino CFD, Machado NC, Cardoso VA, Ribeiro S. Promoção de saúde em unidades básicas: análise das representações sociais dos usuários sobre a atuação da fisioterapia. Cienc Saude Colet. 2011;16(Suppl 1):957-63. doi: 10.1590/S1413-81232011000700027

3. Moretti PGS, Fedosse E. Núcleos de Apoio à Saúde da Família: impactos nas internações por causas sensíveis à atenção básica. Fisioter Pesqui. 2016;23(3):241-7. doi: 10.1590/1809-2950/14662023032016

4. Goulart FAA. Doenças crônicas não transmissíveis: estratégias de controle e desafios para o sistema de saúde [Internet]. Brasília, DF: Organização Pan-Americana da Saúde, 2011 [cited 2020 Nov 3]. Available from: https://www.nescon.medicina. ufmg.br/biblioteca/imagem/4857.pdf

5. Malta DC, Silva MMA, Albuquerque GM, Amorim RCA, Rodrigues GBA, Silva TS, et al. Política Nacional de Promoção da Saúde, descrição da implementação do eixo atividade física e práticas corporais, 2006 a 2014. Rev Bras Ativ Fis Saude. 2014;19(3):286-99. doi: 10.12820/rbafs.v.19n3p286

6. Gomes KV, Zazá DC. Motivos de adesão a prática de atividade física em idosas. Rev Bras Ativ Fis Saude. 2009;14(2):132-38. doi: 10.12820/rbafs.v.14n2p132-138

7. Hirayama MS. Atividade física e doença de Parkinson: mudança de comportamento, auto-eficácia e barreiras percebidas [master's thesis on the Internet]. Rio Claro: Universidade Estadual Paulista; 2006 [cited 2020 Nov 3]. Available from: https://200.145.6.238/bitstream/handle/11449/87372

8. Pisters MF, Veenhof C, Schellevis FG, Twisk JW, Dekker J, De Bakker DH. Exercise adherence improving long term patient 
outcome in patients with osteoarthritis of the hip and/or knee. Arthritis Care Res. 2010;62(8):1087-94. doi: 10.1002/acr.20182

9. Henry KD, Rosemond C, Eckert LB. Effect of number of home exercises on compliance and performance in adults over 65 years of age. Phys Ther. 1999;79(3):270-7. doi: 10078770/ pt.793270

10. Yang D, Liu B, Sun Y, Snetselaar LG, Wallace RB, Bao W. Trends in Adherence to the Physical Activity Guidelines for Americans for Aerobic Activity and Time Spent on Sedentary Behavior Among US Adults, 2007 to 2016. JAMA Netw Open. 2019;2(7):1-11. doi: 10.1001/jamanetworkopen.2019.7597

11. Medina-Mirapeix F, Escolar-Reina P, Gascón-Cánovas JJ, Montilla-Herrador J, Collins SM. Personal characteristics influencing patients adherence to home exercise during chronic pain: a qualitative study. J Rehabil Med. 2009;41(5):347-52. doi: 10.2340/16501977-0338

12. Picorelli AMA, Pereira DS, Felício DC, Gomes DA, Dias RC, Pereira LSM. Adesão de idosas a um programa de exercícios domiciliares pós-treinamento ambulatorial. Fisioter Pesqui. 2015;22(3):291-308. doi: 10.590/1809-2950/13997522032015

13. Monteiro, MC. Adesão de idosos a programa de atividade física em unidade de atenção primária à saúde [master's thesis]. Botucatu: Universidade Estadual Paulista; 2014.

14. Ribeiro JAB, Cavalli AS, Cavalli MO, Pogorzelski LV, Prestes MR, Ricardo LIC. Adesão de idosos a programas de atividade física: motivação e significância. Rev Bras Cienc Esporte. 2012;34(4):969-84. doi: 10.1590/S0101-32892012000400012

15. Benedetti TR, Mazo GZ, Borges LJ. Condições de saúde e nível de atividade física em idosos participantes e não participantes de grupos de convivência de Florianópolis. Cien Saude Colet. 2012;17(8):2087-93. doi: 10.1590/S1413-81232012000800019

16. Mazo GZ, Meuer ST, Benedetti TRB. Motivação de idosos para a adesão a um programa de exercícios físicos. Psicol Am Lat [Internet]. 2009 [cited 2020 Nov 3];18:15-9. Available from: https://pepsic.bvsalud.org/scielo.php?script=sci_arttext\&pid= S1870350X20090002000

17. Mazo GZ, Cardoso FL, Aguiar DD. Programa de hidroginástica para idosos: motivação, auto-estima e auto-imagem. Rev Bras Cineantropom Desempenho Hum. 2006;8(2):67-72.

18. Alves RF, Silva PR, Ernesto MV, Lima AGB, Souza FM. Gênero e saúde: O cuidar do homem em debate. Psicol Teor Prat [Internet]. 2011 [cited 2020 Nov 3];13(3):52-166. Available from: http://pepsic.bvsalud.org/pdf/ptp/v13n3/v13n3a12.pdf

19. Sant'Ana CA, Justino BL, Zarpellon MG, Bertoldo BT, Kuhnen AP. Fatores influentes na desistência de idosos em um programa de exercício físico. Movimento. 2008;14(1):225-39. doi: 10.22456/1982-8918.2303

20. Andreotti M, Okuma S. Perfil sócio-demográfico e de adesão inicial de idosos ingressantes em um programa de educação física. Rev Paul Educ Fis [Internet]. 2003 [cited 2020 Nov 3];17(2):142-53 Available from: http://citrus.uspnet.usp.br/ uploads/arquivo/v17\%20n2\%20artigo6.pdf

21. Saba FKF. Aderência: à prática do exercício físico em academias. São Paulo: Manole; 2001.

22. Carvalho MP, Luckow ELT, Peres W, Lima GG, Siqueira FCV. O envelhecimento e seus fatores de risco associados. RBCEH. 2011;8(2);265-71. doi: 10.5335/rbceh.2012.1296 